\title{
Effect of a pharmacy comprehensive chronic diseases care plan on use of lipid-lowering drugs among patients with hypertension
}

Arsène Zongo, PhD; Scot Simpson, BSP, PharmD, MSc; Jeffrey A Johnson, PhD; and Dean T Eurich, PhD

\section{What is already known about this subject}

- Poor adherence to medications is common among patients with hypertension and has major clinical and economic effects.

- Pharmacist-led interventions have shown inconsistent results in improving adherence.

\section{What this study adds}

- This cohort study assessed the effect of a pharmacist-specific chronic diseases management incentive plan on adherence to lipid-lowering drugs among patients with hypertension.

- The plan had modest effect in improving adherence to lipid-lowering drugs among patients who had poor adherence before receiving the intervention.

- Among patients with good adherence at baseline, the plan was not effective in maintaining good adherence compared with controls.

\author{
Author affiliations \\ Arsène Zongo, PhD, School of Public Health, \\ University of Alberta, Edmonton, Canada; \\ Faculty of Pharmacy, Université Laval, \\ Quebec City, Canada; and Population Health \\ and Optimal Health Practices Research \\ Unit, CHU de Québec, Université Laval \\ Research Centre, Quebec City, Canada. \\ Scot Simpson, BSP, PharmD, MSc, Faculty \\ of Pharmacy and Pharmaceutical Sciences, \\ University of Alberta, Edmonton, Canada. \\ Jeffrey A Johnson, PhD, and Dean T Eurich, \\ PhD, School of Public Health, University of \\ Alberta, Edmonton, Canada.
}

AUTHOR CORRESPONDENCE: Arsène Zongo, 418.682.7511 \#81971; arsene.zongo@pha.ulaval.ca

J Manag Care Spec Pharm. 2021;27(4):426-34

Copyright $(2021$, Academy of Managed Care Pharmacy. All rights reserved.

who had a pre-CACP PDC $<0.80$ were more likely to increase their PDC compared with controls (44.7\% vs. $37.8 \%$; adjusted odds ratio $[\mathrm{aOR}]=1.34 ; 95 \% \mathrm{Cl}=1.22-1.46)$. Conversely, CACP and control patients with a pre-CACP $P D C \geq 0.80$ had similar likelihood to decrease their PDC ( $13.4 \%$ vs. $14.1 \%$; aOR $=0.96 ; 95 \%$ $\mathrm{Cl}=0.88-1.04)$.

CONCLUSIONS: The pharmacy CACP was associated with a modest improvement of adherence to LLD. The incentive system for improved care seemed more effective among patients who had low baseline adherence rates with minimal effect in those with previous good adherence. 
The management of chronic diseases is a continuing challenge for health care systems and patients because of the complexity of the diseases, the frequent coexistence of diseases, the increasing resources needs to manage the growing burden, and the many strategies and interventions required to achieve successful results. ${ }^{1}$ Therefore, the management of chronic diseases remains unsatisfactory for many patients, and the World Health Organization has urged immediate action to better manage chronic diseases around the world. ${ }^{1}$

Cardiovascular diseases are among the most common chronic diseases worldwide and are a major cause of disability and death in the world. ${ }^{2}$ In 2015 , there were an estimated 422.7 million cases of cardiovascular disease and 17.9 million deaths worldwide. ${ }^{2}$ It is well established that hypertension and dyslipidemia are the 2 major contributing risk factors for cardiovascular disease. Dyslipidemia often coexists with hypertension, and the coexistence of the 2 risk factors has more than an additive adverse effect on the risk of cardiovascular disease. ${ }^{3}$ Therefore, beyond the control of blood pressure, the control of the blood lipid profile is necessary to minimize the risk of cardiovascular disease among patients with hypertension.

The pharmacologic management of dyslipidemia is based on the prescription of lipid-lowering drugs. ${ }^{4}$ The role of lipid-lowering drugs in reducing the risk of mortality and morbidity from cardiovascular disease is well established. ${ }^{4,5}$ However, poor adherence to these drugs is very common. ${ }^{6,7}$ Indeed, evidence suggests that between $40 \%$ and $75 \%$ of patients discontinue their statin therapy within 1 year after initiation. ${ }^{7}$ Failure to appropriately use lipid-lowering drugs can thus increase the risk of morbidity and mortality. ${ }^{8,9}$

Following the introduction of a comprehensive care plan for the management of chronic diseases by physicians in 2008, the government of Alberta (Canada) introduced in 2012 a pharmacist-specific chronic diseases management incentive program. ${ }^{10}$ This compensation plan for pharmacy services aimed to encourage Alberta's pharmacists to offer clinical pharmacy services to patients with chronic conditions through comprehensive management strategies. ${ }^{10}$ One element of this plan financially incentivized the codevelopment of a Comprehensive Annual Care Plan (CACP) by pharmacists with patients. To our knowledge, no studies have been specially completed looking at the potential effect of CACP by pharmacists.

Given an overarching aim of the pharmacist CACPs to ensure safe and appropriate use of drugs and monitoring and management of medications, we conducted the present analysis to specifically assess the effect of the pharmacy CACP on adherence to lipid-lowering drugs among beneficiaries who had hypertension. Hypertension was the condition primarily focused on, with over $80 \%$ of all patients receiving a CACP claim in the province based on a diagnosis of hypertension. The expected outcomes of CACP were to improve patient care, proper medication management, and better health outcomes. ${ }^{10}$ We therefore hypothesized that adherence to lipid-lowering drugs would increase as an effect of the plan.

\section{Methods}

\section{STUDY DESIGN}

We conducted a population-based cohort study of patients who received a pharmacy CACP between 2012 and 2015 (intervention group). A control group consisted of patients who qualified to receive the CACP but did not receive it at the moment they were selected as controls (see the Study Population section for further details). We then compared adherence in the year before the CACP date with the year after.

\section{DESCRIPTION OF THE CACP PLAN}

The CACP is a single written document that includes data from the patient's medical information, current therapies, health challenges, information about other health care providers involved in the patient's care, and other relevant information that may affect the patient's health or treatment options. The CACP is prepared in collaboration with the patient so that it can take into account the patient's values and personal health goals. The CACP serves to help patients better understand the management of their complex medical conditions, to assist patients in navigating through the health care system, to improve patient access to the team of health care professionals, and to serve as a self-management tool to help patients create short- to longterm goals as they manage their chronic health conditions. Once completed, the CACP is signed by the patient and a pharmacist.

Patients were eligible for a CACP if they had 2 or more of the following conditions: asthma, diabetes, chronic obstructive pulmonary disease, heart disease, heart failure, or hypertension. Alternatively, patients could qualify if they had 1 of the above conditions combined with obesity, mental health problems, addiction disorders, or tobacco use.

Components of the pharmacy CACP include administration of injections, assessment and modifying prescriptions based on individual patient needs, prescription renewals, assessment of prescriptions for emergency needs, assessment for initiating medication therapy, medication assessments to ensure safe and appropriate use such as refusal to fill a prescription or a trial prescription, tobacco 
cessation services, standard medication management assessment for diabetics, and administration of publicly funded vaccines, such as seasonal influenza. ${ }^{10}$

\section{SOURCES OF DATA}

We used provincial health care administrative databases and vital statistics files provided by Alberta Health. More specifically, we combined databases from Alberta Services (i.e., Population and Vital Statistic Data); Alberta Health (Discharge Abstract Database, Ambulatory Care Classification Database, and Alberta Physician Claims database); and Alberta Blue Cross/PIN (Medications database). The Medications database contains information for drug class, Anatomical Therapeutic Chemical codes, name, generic and brand name, strength and dosage, date of dispensation, quantities dispensed, and the number of days covered by the dispensed drugs. The prescriber, the dispensed pharmacy, and costs were also recorded. This database captures $95 \%$ of all dispensed drugs, irrespective of age. Accuracy and validity are routinely checked through computerized processing.

The Population and Vital Statistic data contains information on patient sex, age, marital status, immigration and emigration data, date of death, and cause of death according to the World Health Organization algorithm using International Classification of Diseases (ICD) codes. The Discharge Abstract Database contains all hospital services, length of stay, diagnosis (with ICD codes), and procedure intervention. The Alberta Physician Claims Database contains the date of service, ICD code associated with the claim, procedure and billing information, and the specialty of the billing physician. Data and coding accuracy is routinely validated, both provincially and centrally. All databases were linked at the patient level based on personal health number.

\section{STUDY POPULATION}

The source population of the current study was made of all patients who received a CACP between 2012 and 2015 $(\mathrm{N}=149,877)$. For the purpose of assessing the overall effectiveness of the CACP for various outcomes, each of the 149,877 CACP patients was matched to up to 2 controls. Controls were patients who qualified to receive the $\mathrm{CACP}$ but did not receive it at the moment they were selected as controls. Controls were matched to each CACP patient based on age ( \pm 2 years), sex, service provider (same pharmacy), similar date of service in the same pharmacy $( \pm 6$ months) and same CACP-qualifying conditions based on a 5 -year history within the administrative data. The service date for the controls was considered as a pseudo CACP date for simplication.
From the base-matched cohort, we selected all patients who had hypertension-individuals with ICD-9 codes (401.x) or ICD-10 codes (I11.x). Patients who received the CACP and the controls were required to have at least 1 claim for any lipid-lowering drug in the period -730 to -365 days before the CACP date (index period or pseudo-index period for the controls). The requirement for patients to have a lipid-lowering drug in the index period was to ensure that we were comparing adherence levels for patients fully established on lipid-lowering drugs, as opposed to new users, where primary adherence failure could occur. ${ }^{6}$

To ensure comparability of groups, we excluded patients who died or who were not covered by a provincial drug plan (i.e., those who moved out of the province) during the year following the date of CACP. This was to ensure that financial or length of follow-up issues would not affect our results (Figure 1).

\section{OUTCOMES}

The outcome of this study was change in adherence to lipidlowering drugs in the year following the CACP. Medication adherence was defined as the proportion of days covered (PDC) by any lipid-lowering drug based on dispensing dates and days supply (i.e., total number of days covered by drugs/ total days in the period). Two PDCs were computed for each patient: one in the 365 days before the CACP (pre-CACP PDC) and another one in the 365 days after the CACP (postCACP PDC). Because the Medications database does not include dispensation records for medications used during hospitalization, each PDC was adjusted for patient-specific hospital days that occurred during the specified period of adherence (i.e., hospital days were subtracted from the denominators). ${ }^{11}$

To assess changes in PDC, we split the sample into 2 groups according to the pre-CACP PDC (i.e., patients with pre-CACP $\mathrm{PDC}<0.80$ [poor adherence] and patients with pre-CACP $\mathrm{PDC} \geq 0.80$ [good adherence]). We then defined change as the increase or decrease of post-CACP PDC by 0.20 , respectively, in the groups of patients with pre-CACP $\mathrm{PDC}<0.80$ and $\geq 0.80$. This value of 0.20 was based on evidence suggesting that an increase in claimbased adherence by $20 \%$ is associated with net savings in all-cause health care costs for diabetes, hypertension, and hypercholesterolemia. ${ }^{12}$

\section{OTHER VARIABLES}

In addition to the initial matching variables (i.e., age, gender, pharmacy, and CACP-qualifying conditions), we also considered variables related to drug-induced or toxic myopathy (as a proxy for statin-related myopathy), chronic kidney disease, the area of residence (rural vs. urban), and health care 


\section{FIGURE 1 Selection of the Study Population}

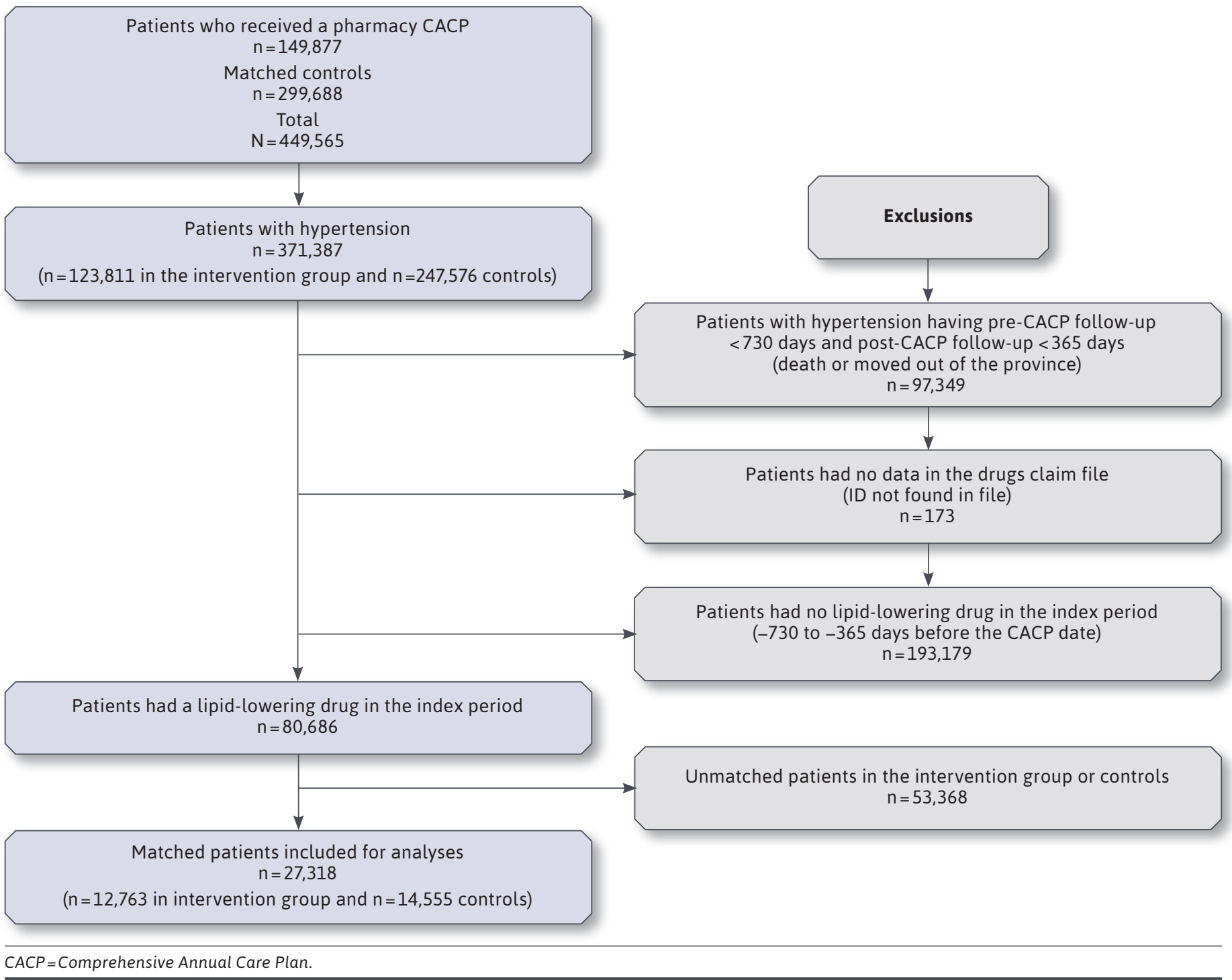

resource use in each period (i.e., the total number of hospital stays and visits to physician), as well as use of other prescription medications (i.e., total number of prescribed drugs in each period).

\section{DATA ANALYSIS}

We used descriptive statistics to describe the overall characteristics of the study population, stratifying according to the group (CACP or control group). We calculated the median value of PDCs and the proportion of patients with good adherence $(\mathrm{PDC} \geq 0.80)$ in each period, as well as the proportion of patients who increased or decreased their adherence after the CACP.

In the sample of patients with pre-CACP $\mathrm{PDC}<0.80$, we performed univariate and multivariate logistic regressions to assess the probability of increasing the post-CACP PDC by $\geq 0.20$. In distinct logistic regression models, we also assessed the probability that patients with good pre-CACP adherence (i.e., $\geq 0.80$ ), decrease their post-CACP PDC by $\geq 0.20$. Because splitting the sample into good and poor 


\section{TABLE 1 Characteristics and Estimates of Adherence to Lipid-Lowering Drugs of Patients with Hypertension Who Received a Pharmacy CACP and Controls $(\mathrm{N}=27,318)$}

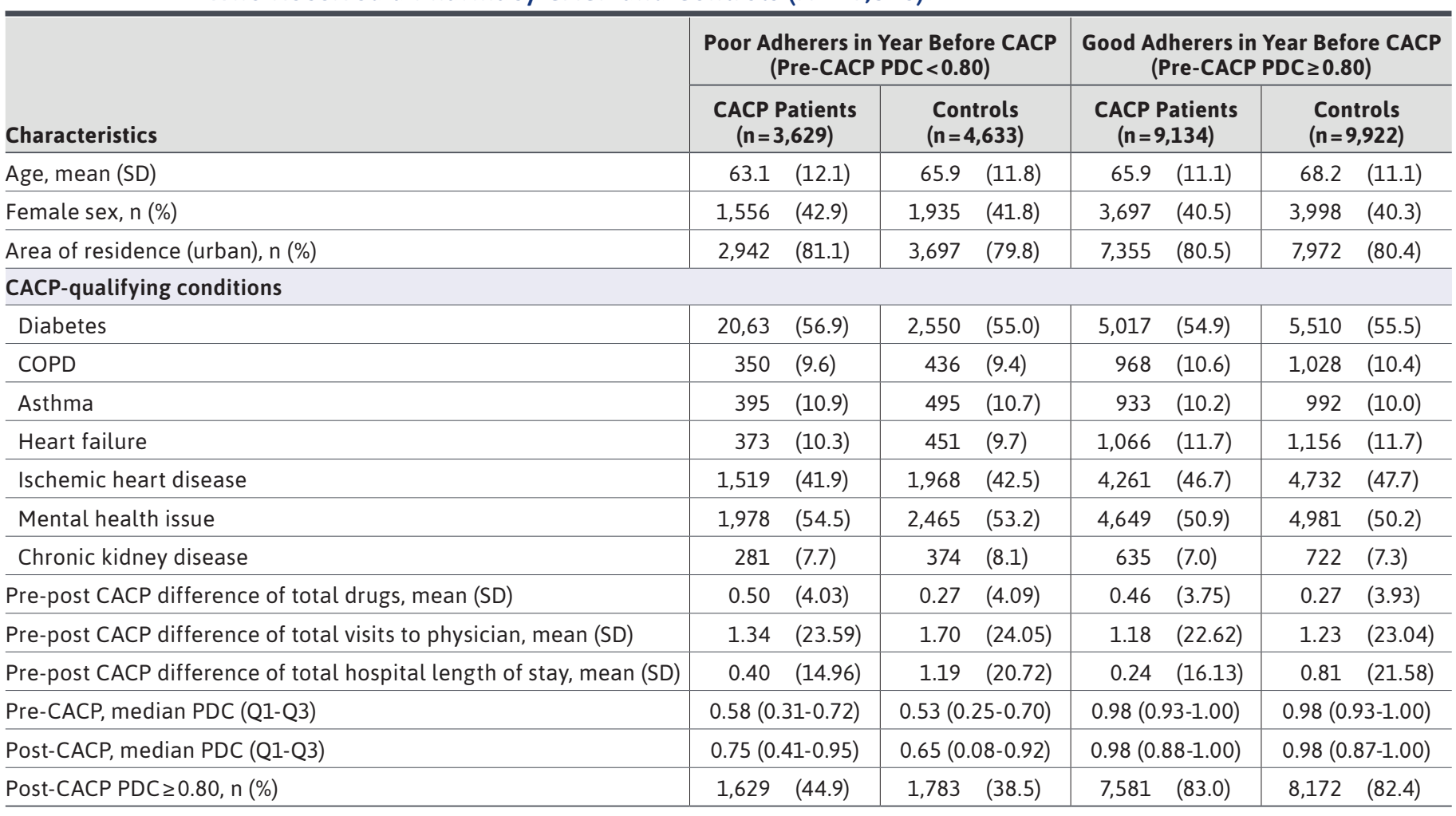

Note: Pre- and post-CACP variables were measured in the year before and the year after the CACP, respectively.

$C A C P=$ Comprehensive Annual Care Plan; $C O P D=$ chronic obstructive pulmonary disease; $P D C=$ proportion of days covered by lipid-lowering drugs.

adherers had distorted the initial matching, all matching variables and the other variables previously described were considered to adjust each regression model. For the same reason, we used nonconditional logistic regression to analyse the data instead of a conditional logistic regression required for a matched design.

In sensitivity analyses, we repeated all regression analyses by changing the definition of good adherence to 0.70 or 0.90 , as well as changing the increase or decrease in PDC of only $5 \%$ or $10 \%$ (as opposed to $20 \%$ in the base case).

The analyses were carried out using SAS version 9.4 (SAS Institute, Cary, NC). This study was approved by the University of Alberta Human Research and Ethics Board (Pro00083926). All data were fully anonymized by Alberta Health before sent to the researchers. Patient consent was not required.

\section{Results}

Among CACP patients $(\mathrm{n}=149,877)$ and the controls $(\mathrm{n}=299,688), 371,387 \quad(82.6 \%)$ had hypertension. Among them, 27,318 (12,763 CACP patients and 14,555 controls) fulfilled the inclusion criteria and were considered for analyses (Figure 1). The mean (SD) age of patients was 66.39 (11.47), and $41 \%$ of the sample were women (Table 1).

Considering the median values and proportion of patients with a PDC $\geq 0.80$, adherence to lipid-lowering drugs in each group were similar in the 2 periods (Table 1). Overall, $71 \%$ of CACP patients had a $\mathrm{PDC} \geq 0.80$ in the pre-CACP period, compared with $67 \%$ of controls (Table 2 ).

When looking at change in the PDC, CACP patients who had a pre-CACP $\mathrm{PDC}<0.80$ were more likely to increase their PDC by $20 \%$ compared with controls ( $44.7 \%$ vs. $37.8 \%$; adjusted odds ratio $[\mathrm{aOR}]=1.34,95 \% \mathrm{CI}=1.22-1.46$; Table 3 ). Conversely, $\mathrm{CACP}$ and control patients with a pre-CACP 


\section{TABLE 2}

\section{Change in Adherence to Lipid-Lowering Drugs According to Pre-CACP Adherence Dichotomized} as $P D C \geq 0.80$

\begin{tabular}{|c|c|c|c|c|c|c|c|c|}
\hline \multirow{2}{*}{$\begin{array}{l}\text { Change in } \\
\text { Post-CACP PDC }\end{array}$} & \multicolumn{3}{|c|}{ Subsample with Pre-CACP PDC $<0.80, n(\%)$} & \multicolumn{5}{|c|}{ Subsample with Pre-CACP PDC $\geq 0.80, n(\%)$} \\
\hline & $\operatorname{CACP}(n=3,629)$ & Controls $(n=4,633)$ & Total $(n=8,262)$ & $\operatorname{CACP}(n=9,134)$ & Controls ( & $(n=9,922)$ & Total (n & $1=19,056)$ \\
\hline Post-CACP PDC $\geq 0.80$ & $1,629(44.9)$ & $1,783 \quad(38.5)$ & $3,412 \quad(41.3)$ & $7,581 \quad(83.0)$ & 8,172 & $(82.4)$ & 15,753 & $(82.7)$ \\
\hline Post-CACP PDC $<0.80$ & $2,000 \quad(55.1)$ & $2,850 \quad(61.5)$ & $4,850 \quad(58.7)$ & $1,553 \quad(17.0)$ & 1,750 & $(17.6)$ & 3,303 & $(17.3)$ \\
\hline $\begin{array}{l}\text { Post-CACP PDC } \\
\text { increase by } \geq 0.20\end{array}$ & $1,622(44.7)$ & 1,752 & $3,374 \quad(40.8)$ & - & & - & & - \\
\hline $\begin{array}{l}\text { Post-CACP PDC } \\
\text { increase by } \geq 0.10\end{array}$ & $2,093 \quad(57.7)$ & $2,223(48.0)$ & $4,316 \quad(52.2)$ & - & & - & & - \\
\hline $\begin{array}{l}\text { Post-CACP PDC } \\
\text { increase by } \geq 0.05\end{array}$ & $2,255 \quad(62.1)$ & $2,413 \quad(52.1)$ & $4,668 \quad(56.5)$ & - & & - & & - \\
\hline $\begin{array}{l}\text { Post-CACP PDC } \\
\text { decrease by } \geq 0.20\end{array}$ & - & - & - & $1,222(13.4)$ & 1,397 & (14.1) & 2,617 & $(13.7)$ \\
\hline $\begin{array}{l}\text { Post-CACP PDC } \\
\text { decrease by } \geq 0.10\end{array}$ & - & - & - & $1,878 \quad(20.6)$ & 2,142 & $(21.6)$ & 4,020 & $(21.1)$ \\
\hline $\begin{array}{l}\text { Post-CACP PDC } \\
\text { decrease by } \geq 0.05\end{array}$ & - & - & - & $2,497 \quad(27.3)$ & 2,826 & $(28.5)$ & 5,323 & $(27.9)$ \\
\hline
\end{tabular}

$C A C P=$ Comprehensive Annual Care Plan; $P D C=$ proportion of days covered by any lipid-lowering drug.

PDC $\geq 0.80$ had similar likelihood to decrease their PDC (13.4\% vs. $14.1 \%$; aOR=0.96, 95\% CI=0.88-1.04; Table 3 ).

Sensitivity analyses using a PDC cut-point of 0.70 or 0.90 , as well as sensitivity analyses using an increase or decrease of only $5 \%$ or $10 \%$ in PDC, showed similar trends (Supplementary Tables 1 and 2, available in online article).

\section{Discussion}

The pharmacy CACP compensation plan was implemented to improve and incentivize chronic disease management by pharmacists. Our results suggest that compared with usual care, modest improvements in adherence to lipid-lowering drugs were observed for patients receiving a CACP. Patients who initially had poor pre-CACP adherence to lipid-lowering drugs and were managed under the pharmacy CACP plan were moderately more likely to increase their adherence to lipid-lowering drugs. However, among patients who had good pre-CACP adherence, approximately $14 \%$ had substantial declines in adherence in the post-CACP period, which was similar between those who received the CACP and those who did not receive it. Thus, the overall clinical utility of the CACPs is difficult to qualify, since, although some improvements were noted in those with poor adherence, CACPs had no effect in maintaining good adherence in the year following implementation in those whose adherence was initially good.
A possible explanation for these results may be that medication adherence is a complex behavior that requires the combination of multiple intensive approaches (e.g., behavioral intervention, motivational interview, patient education and information, and reminders) to obtain significant changes. ${ }^{13}$ Therefore, the lack of some, or all, of these specific adherence-enhancing intervention components in the pharmacy CACP plan may explain the modest effect of the plan in improving or maintaining good adherence for the majority of patients. Given the importance of adherence to lipid-lowering drugs in reducing the risk of cardiovascular complications, the introduction of such components in the plan may help patients to benefit more from the effect of the drugs. The assessment of barriers, the views, and the satisfaction of patients and pharmacists regarding the importance of the plan in the long-term use of drugs could also provide some explanations for the modest improvement of adherence to lipid-lowering drugs.

The similar reduction in adherence among all (CACP and control) patients with good pre-CACP adherence may also simply reflect the phenomenon of regression to the mean..$^{14}$ This phenomenon must always be considered in the interpretation of changes observed in groups that are nonrandomly selected on the basis of the degree of some baseline characteristic. In nonrandomized studies such as ours, comparison with a control group is a remedy to control for regression to the mean. As such, given the differential change observed in the CACP versus control 


\section{TABLE 3}

\section{Effect of Pharmacy CACP on Adherence to Lipid}

\section{Unadjusted Odds Ratio $(95 \% \mathrm{Cl}) \quad$ Adjusted Odds Ratio $(95 \% \mathrm{Cl})$}

PDC increased by 0.20 (CACP vs. controls) $^{a}$

\begin{tabular}{l|l|l}
\hline Model 1: Subsample with pre-CACP PDC $<0.80(\mathrm{n}=8,262)$ & $1.33(1.22-1.45)$ & $1.34(1.22-1.46)$ \\
\hline Model 2: Subsample with pre-CACP PDC $<0.90(\mathrm{n}=11,415)$ & $1.22(1.13-1.32)$ & $1.22(1.12-1.32)$ \\
\hline Model 3: Subsample with pre-CACP PDC $<0.70(\mathrm{n}=6,032)$ & $1.45(1.31-1.61)$ & $1.43(1.29-1.59)$ \\
\hline PDC decreased by 0.20 (CACP vs. controls) & \multicolumn{3}{|c}{} \\
\hline Model 1: Subsample with pre-CACP PDC $\geq 0.80(\mathrm{n}=19,056)$ & $0.94(0.87-1.02)$ & $0.96(0.88-1.04)$ \\
\hline Model 2: Subsample with pre-CACP PDC $\geq 0.90(\mathrm{n}=15,903)$ & $0.95(0.86-1.04)$ & $0.96(0.88-1.06)$ \\
\hline Model 3: Subsample with pre-CACP PDC $\geq 0.70(\mathrm{n}=21,286)$ & $0.92(0.85-1.00)$ & $0.93(0.86-1.01)$ \\
\hline
\end{tabular}

${ }^{a}$ Adjusted for post- vs. pre-CACP difference of total drugs, difference of hospital length of stay, difference of total visits to physicians, area of residence (urban vs. rural), age, gender, diabetes, ischemic heart disease, mental health issue, asthma, and COPD.

$C A C P=$ Comprehensive Annual Care Plan; $C O P D=$ chronic obstructive pulmonary disease; $P D C=$ proportion of days covered by any lipid-lowering drug .

patients with poor pre-CACP, we are more confident that it is a result of the intervention.

We were not aware of studies that assessed the effect of similar incentive initiatives targeting pharmacists on medication adherence. However, in a 6-month randomized controlled trial comparing pay-for-performance (P4P) and fee-for-service (FFS) initiatives for Alberta pharmacists on blood pressure reduction in hypertensive patients, there was no statistically significant difference in blood pressure reduction between those patients randomized to $\mathrm{P} 4 \mathrm{P}$ and FFS remuneration. ${ }^{15}$

In general, pharmacy-led interventions aimed specifically at improving adherence to lipid-lowering drugs have demonstrated inconsistent findings of their efficacy. ${ }^{16-22}$ Indeed, interventions based on education from pharmacists tailored to identified misconceptions and beliefs about patient medication ( 3 contacts with each patient) ${ }^{19}$ pharmacist-delivered telephone counseling calls (5 contacts), ${ }^{17}$ and a single automated phone call from a pharmacist to remind patients of the initiation of treatment resulted in an absence of improvement of adherence to lipid-lowering drugs..$^{18}$ However, more intensive interventions (i.e., weekly telephone contacts by the same pharmacist for 12 weeks $)^{20}$ or long-term multicomponent pharmacist-led interventions (information, education, and reminders for 12 months) 21,22 $^{21}$ resulted in significant improvement of adherence to lipidlowering drugs. ${ }^{20-22}$ A Cochrane systematic review that pooled the results of these latter studies showed that patients in the interventions group were almost 3 times more likely to improve their adherence compared with patients who received usual care (pooled $\mathrm{OR}=2.87,95 \%$ $\mathrm{CI}=1.91-4.29){ }^{16}$ Collectively, previous research suggests that for a CACP to successfully improve adherence, the CACP must contain multifaceted components targeting adherence and must instill multiple contacts with patients of sufficient intensity and duration around adherence issues if improving adherence to lipid-lowering and overall prescribed drugs is a major objective of the plan.

To incentivize the use and compensate pharmacists for developing CACPs with patients, a pharmacy can receive compensation for initiating a CACP totaling \$125 per year per patient. Importantly, these reimbursements for pharmacist-billed CACPs are paid at the pharmacy level, not to the individual pharmacist. Although the CACP program has been operationalized for several years with relatively rapid uptake, no formal economic evaluation has occurred beyond reports of the total expenditures.

Among the challenges to such an assessment includes determining the scope of an economic evaluation because the CACP program can have a wide range of effects on patients or the health system as a whole. For example, the CACP program may result in overall improvements in chronic disease care, satisfaction with services, changes in the use of other drugs or health services which may be more difficult to capture and value.

Moreover, it is also possible that the full effect of the CACP program may not be observed for many years. Thus, although the costs of the program are relatively easy to identify, the potential economic benefits of the program are more difficult to quantify. Nonetheless, an economic model should be attempted to ensure that value is being delivered within our publicly funded health care system. 


\section{STRENGTHS AND LIMITATIONS}

Strengths of our study include analysis of all CACP completed in hypertensive patients since the program was initiated in the province, the large population-based size of the sample, the use of a control group, and accounting for the trends in adherence before the CACP.

Our study is not without limitations, however. First, we assessed adherence to lipid-lowering drugs using drug claims data, assuming that drugs filled by patients were appropriately used. This may overestimate adherence estimates for patients who partially took their filled drugs or never consumed them. However, the overestimation of adherence is likely to affect patients in the CACP group and controls in a similar manner, leading to possible nondifferential information bias of adherence.

Second, some unmeasured confounders, such as perceived adverse events of drugs and patient literacy, were not available in the claims data and therefore could not be accounted for. For example, myopathy is a frequent adverse event of statins, affecting $1 \%-25 \%$ of users and is one of the main causes of poor adherence to statin therapy. ${ }^{23,24}$ Our attempt to include a proxy variable for toxic myopathy induced by lipid-lowering drugs in our analyses was inconclusive. However, as we computed adherence to any lipid-lowering drug, patients were allowed to switch from one statin to another statin or to other lipid-lowering drugs and possibly minimized the effect of those adverse events in our estimates. Moreover, all patients were persistent users of statins for up to 2 years before the CACP; as a result, changes or discontinuations of statins due to adverse events should be minimal.

The complexity of the overall drugs regimen is also an important factor affecting medication adherence and may not have been fully captured in our analyses. ${ }^{13}$ Indeed, although we used the total number of drugs as a proxy for the complexity of drug regimens, this approach did not account for routes and time of administration and relation of drugs with food intake. Importantly, however, both groups of patients had nearly identical levels of medication burden, so substantial differences would not have been expected.

Finally, the approach we used to analyse the data (i.e., by splitting the sample into good and poor adherers according to the pre-CACP adherence) did not preserve the initial matching (i.e., we lost the advantage of the matching). However, because many patients had perfect or very good adherence in the year before the CACP (i.e., a pre-CACP $\mathrm{PDC}=1$ or $\geq 0.80$ ), comparing the pre- and post-CACP PDCs for the whole sample would have introduced a bias. Indeed, there is no room for further improvement for patients with a pre-CACP $\mathrm{PDC}=1$.

\section{Conclusions}

The pharmacy CACP plan was associated with a modest improvement of adherence to lipid-lowering drugs among patients with hypertension. The incentive system for improved care seemed more effective among patients who had low baseline adherence rates with minimal effect on maintaining adherence in those presenting with overall good adherence before the CACP. To obtain substantial improvements in adherence, the introduction and incentivising of specific interventions targeting medication adherence may be warranted.

\section{DISCLOSURES}

This work was supported by a grant from the Institute of Health Economics, with funding from Alberta Innovates and Eli Lilly Canada. The sponsor had no role in the study design, data acquisition, analysis, interpretation of the results, and the decision to publish. The authors have no conflicts of interest to disclose.

This study is based on data provided by Alberta Health. The interpretation and conclusions contained herein are those of the researchers and do not necessarily represent the views of the government of Alberta nor the funder (Institute of Health Economics). Neither the government nor Alberta Health nor the Institute of Health Economics express any opinion in relation to this study.

\section{REFERENCES}

1. World Health Organization. Preventing chronic diseases: a vital investment. 2005. Accessed February 22, 2021. https://www. who.int/chp/chronic_disease_report/ contents/en/

2. Roth GA, Johnson C, Abajobir A, et al. Global, regional, and national burden of cardiovascular diseases for 10 causes, 1990 to 2015. J Am Coll Cardiol. 2017;70(1):1-25.

3. Dalal JJ, Padmanabhan TN, Jain P, Patil S, Vasnawala H, Gulati A. LIPITENSION: interplay between dyslipidemia and hypertension. Indian J Endocrinol Metab. 2012;16(2):240-45.

4. Ewang-Emukowhate M, Wierzbicki AS. Lipid-lowering agents. J Cardiovasc Pharmacol Ther. 2013;18(5):401-11.

5. Cholesterol Treatment Trialists Collaboration, Baigent C, Blackwell L, Emberson J, Holland LE, Reith C, et al. Efficacy and safety of more intensive lowering of LDL cholesterol: a meta-analysis of data from 170,000 participants in 26 randomised trials. Lancet. 2010;376(9753):1670-81.

6. Ho PM, Bryson CL, Rumsfeld JS. Medication adherence: its importance in cardiovascular outcomes. Circulation. 2009;119(23):3028-35. 
7. Banach M, Stulc T, Dent R, Toth PP. Statin non-adherence and residual cardiovascular risk: There is need for substantial improvement. Int J Cardiol. 2016;225:184-96.

8. Karlsson SA, Hero C, Svensson AM, et al. Association between refill adherence to lipid-lowering medications and the risk of cardiovascular disease and mortality in Swedish patients with type 2 diabetes mellitus: a nationwide cohort study. BMJ Open. 2018;8(3):e020309.

9. Rodriguez F, Maron DJ, Knowles JW, Virani SS, Lin S, Heidenreich PA.

Association of statin adherence with mortality in patients with atherosclerotic cardiovascular disease. JAMA Cardiol. 2019;4(3):206-13.

10. Alberta Health. Health Benefits and Compliance. Compensation for Pharmacy Services. 2014. Accessed March 2, 2021. https://open.alberta.ca/ publications/compensation-plan-forpharmacy-services

11. Dong YH, Choudhry NK, Krumme A, et al. Impact of hospitalization on medication adherence estimation in claims data. J Clin Pharm Ther. 2017;42(3):318-28.

12. Sokol MC, McGuigan KA, Verbrugge RR, Epstein RS. Impact of medication adherence on hospitalization risk and healthcare cost. Med Care. 2005;43(6):521-30.
13. World Health Organization. Adherence to long-term therapies: evidence for action. 2003. Accessed February 22, 2021. http://www.who.int/chp/knowledge/ publications/adherence_report/en/

14. Chiolero A, Paradis G, Rich B, Hanley JA. Assessing the relationship between the baseline value of a continuous variable and subsequent change over time. Front Public Health. 2013;1:29.

15. Houle SK, Charrois TL, McAlister FA, et al. Pay-for-performance remuneration for pharmacist prescribers' management of hypertension: a substudy of the RxACTION trial. Can Pharm J (Ott). 2016;149(6):345-51.

16. van Driel ML, Morledge MD, Ulep R, Shaffer JP, Davies P, Deichmann R. Interventions to improve adherence to lipid-lowering medication. Cochrane Database Syst Rev. 2016;12:CD004371.

17. Ma Y, Ockene IS, Rosal MC, Merriam PA, Ockene JK, Gandhi PJ. Randomized trial of a pharmacistdelivered intervention for improving lipid-lowering medication adherence among patients with coronary heart disease. Cholesterol. 2010;2010:383281.

18. Fischer MA, Choudhry NK, Bykov K, et al. Pharmacy-based interventions to reduce primary medication nonadherence to cardiovascular medications. Med Care. 2014;52(12):1050-54.
19. Gujral G, Winckel K, Nissen LM, Cottrell WN. Impact of community pharmacist intervention discussing patients' beliefs to improve medication adherence. Int J Clin Pharm. 2014;36(5):1048-58.

20. Faulkner MA, Wadibia EC, Lucas BD, Hilleman DE. Impact of pharmacy counseling on compliance and effectiveness of combination lipid-lowering therapy in patients undergoing coronary artery revascularization: a randomized, controlled trial. Pharmacotherapy. 2000;20(4):410-16.

21. Ho PM, Lambert-Kerzner A, Carey EP, et al. Multifaceted intervention to improve medication adherence and secondary prevention measures after acute coronary syndrome hospital discharge: a randomized clinical trial. JAMA Intern Med. 2014;174(2):186-93.

22. Vrijens B, Belmans A, Matthys K, de Klerk E, Lesaffre E. Effect of intervention through a pharmaceutical care program on patient adherence with prescribed once-daily atorvastatin. Pharmacoepidemiol Drug Saf. 2006;15(2):115-21.

23. Taylor BA, Thompson PD. Musclerelated side-effects of statins: from mechanisms to evidence-based solutions. Curr Opin Lipidol. 2015;26(3):221-27.

24. Pirillo A, Catapano AL. Statin intolerance: diagnosis and remedies. Curr Cardiol Rep. 2015;17(5):27. 